\title{
Hyperprolactinemia and the Dopamine Receptor
}

\section{Keith A Hansen, \\ a report by}

Department of Obstetrics \& Gynecology, School of Medicine, The University of South Dakota

DOI: $10.17925 /$ USE.2006.00.1.2b

\section{Prolactin}

Prolactin is a polypeptide hormone produced and secreted by the adenohypophysis. This hormone is in the family with growth hormone and human placental lactogen; in fact, they are sufficiently homologous to suggest a common ancestral gene.

Prolactin's primary function in the human is assisting in the development and maturation of the breast during pregnancy and the subsequent production of milk during lactation. Prolactin is a unique anterior pituitary hormone, as its release is under tonic inhibitory control by dopamine. Dopamine is delivered to the adenohypophysis from the hypothalamus via the hypothalamic-hypophyseal portal system. ${ }^{1}$ Dopamine binds to the dopamine D2 receptors on the surface of the lactotroph, which diminish intracellular cyclic AMP (cAMP), consequently decreasing prolactin secretion. Any factor disrupting the delivery of dopamine to the anterior pituitary or disturbing signal transduction may result in hyperprolactinemia. There are a number of agents that also simulate prolactin synthesis and release, including thyrotropin releasing hormone (TRH), vasoactive intestinal peptide, oxytocin, and estrogen. ${ }^{2}$

Hyperprolactinemia

Hyperprolactinemia results in secondary hypogonadism and usually presents in a dimorphic fashion in both females and males. In females, elevated prolactin presents with galactorrhea, irregular or absent menses, infertility, or local symptoms of an expanding pituitary tumor. In males the symptoms are more subtle, or at least more frequently ignored, often resulting in delays in seeking medical care. In males, symptoms include decreased libido, erectile dysfunction, other signs of hypogonadism, and local symptoms from an expanding pituitary tumor..$^{3-5}$

Local symptoms result from an expanding pituitary adenoma in the confined space of the sella turcica. While the tumor expands extra-sellar, it usually moves superiorly where it impinges on the optic nerve resulting in visual changes, particularly in the visual fields. A more invasive, large tumor can involve the cavernous sinus and result in other symptoms including diplopia, ptosis, and facial numbness from involvement of cranial nerves III, IV,VI, and branches of V. One of the most common local symptoms related to the expanding tumor include headaches. If the tumor is large enough that it compresses and interferes with function of the normal pituitary gland, the patient may present with selective pituitary hormone deficiency symptoms or pan-hypopituitarism. ${ }^{6}$

\section{Etiologies of Hyperprolactinemia}

The immunometric assay with monoclonal antibodies against prolactin is a very useful assay for detecting circulating prolactin. There are false negative results when the prolactin levels are markedly elevated due to the hook effect of the antibodies used in the assay. False positive results can occur when the patient has heterophilic antibodies directed against the antibodies used in the assay system. Another cause of falsely abnormal results is when the patient has circulating big (50-60 kilodaltons) and very big prolactin (150 kilodaltons), which is a biologically, relatively inactive variant of the native prolactin molecule. ${ }^{7,8}$

Hyperprolactinemia can result from three major causes including stress-response, pharmacology, and pathologic etiologies. Prolactin is a stress hormone that increases in response to a number of physiologic and stressful conditions. Examples of physiologic conditions include pregnancy, lactation, and eating foods high in amino acids in particular. Stressful situations can also result in elevated prolactin levels including the stress of having blood drawn. A male patient presented with a prolactin level of $153 \mathrm{ng} / \mathrm{mL}$ after a near-syncopal episode during venipuncture. A repeat level in the fasting state, drawn after 30 minutes of rest following placement of a heparin-lock, was normal. This marked elevation in prolactin following venipuncture illustrates the role stress can play and the importance of repeating the level in a fasting, non-stressed state. ${ }^{9}$ 
In patients with hyperprolactinemia one must rule out primary hypothyroidism. In hypothyroidism the decreased feedback of thyroid hormone on the hypothalamus results in an elevated thyrotropin releasing hormone (TRH) level. TRH then binds to the lactotroph and stimulates the release of prolactin. Once the patient's hypothyroidism is treated, the prolactin level normalizes.

There are a number of pharmacologic agents that interfere with the production or action of dopamine. These medications include dopamine receptor blockers, (such as the phenothiazines), dopamine synthesis inhibitors (such as alpha-methyldopa), opioids, serotonin reuptake inhibitors, and estrogens, among others. Interference with dopamine inhibition of the lactotrophs results in an elevated prolactin level. When faced with a patient on medication(s) that interfere with dopamine and an elevated prolactin level, one must perform an evaluation to rule out the pathologic causes of hyperprolactinemia. An option that would reduce one's concern about these patients would be to obtain a prolactin level prior to starting drugs with known dopamine effects. Another alternative would be to discontinue the suspected prolactin-elevating drug and repeat the prolactin level. ${ }^{10}$

Pathologic etiologies of hyperprolactinemia include prolactinomas, adenohypophyseal adenomas, which produce and secrete prolactin, and any other pituitary tumor or lesion that interferes with the transport of dopamine to the lactotrophs. Prolactinomas are one of the most common pituitary tumors and are classified as microadenomas that are less than $10 \mathrm{~mm}$ or macroadenomas that are $10 \mathrm{~mm}$ or greater in diameter. These tumors actively produce and secrete prolactin, usually resulting in levels over $100 \mathrm{ng} / \mathrm{mL}$. Idiopathic hyperprolactinemia results when one has elevated prolactin levels, but a normal imaging study of the pituitary gland. Whether idiopathic hyperprolac-tinemia is due to a microadenoma that is below the sensitivity level of current pituitary imaging studies or hyperplasia was unknown at the time of press. Other lesions, such as a large macroadenoma that interferes or blocks the transmission of dopamine down the pituitary stalk to the lactotroph, will also elevate prolactin levels. Other miscellaneous causes of elevated prolactin levels include anterior chest wall trauma or scarring, which is felt to cause continued afferent stimulation similar to lactation. ${ }^{11,12}$

\section{Evaluation}

The evaluation of hyperprolactinemia initially requires confirmation of the hyperprolactinemic state by repeating the level with a fasting, non-stressed venipuncture. In persistent elevations of prolactin, a thorough history and physical examination should be performed looking for evidence suggesting the etiology. Other important laboratory data include insulin-like growth factor (IGF)-1 to rule out a growth hormone-prolactin co-secreting pituitary adenoma. One also wants to rule out the possibility of multiple endocrine neoplasia type-1 (MEN-1) by taking a thorough family history and obtaining calcium and phosphorous levels, as well as any other indicated blood tests. If there is evidence of a pituitary adenoma or lesion, one may need to evaluate for other pituitary hormone excesses or deficiencies. The next step in evaluating a patient with hyperprolactinemia is to perform imaging of the sella turcica looking for any anatomic abnormalities, including pituitary adenomas. ${ }^{13}$

\section{Molecular Biology}

The molecular biology of idiopathic hyper-prolactinemia and lactotroph adenomas remains uncertain, but an area of research interest. Dopamine D2 receptors are localized to lactotrophs in the adenohypophysis and, when coupled to dopamine, maintain inhibition of prolactin secretion. An attractive hypothesis for the development of prolactinomas would be a molecular defect in the DRD2 or in its secondary messengers releasing the lactotrophs from tonic inhibition.

Dopamine D2 receptors have been localized to the lactotrophs in prolactinomas both in vivo and in vitro. No mutations in the Dopamine D2 receptors have been detected in individuals with prolactin-secreting adenomas. In a subpopulation of prolactinomas that are resistant to dopamine, there is a decrease in DRD2 density on the lactotroph cell surface. These adenomas also have alterations in DRD2 isoforms and Gialpha (Gi)/Goalpha (Go) proteins. ${ }^{14}$ In female DRD2 null mice, hyperprolactinemia with lacotroph hyperplasia occurs at nine to 12 months of age. ${ }^{15}$ At 17 to 20 months of age these DRD2 null mice develop pituitary lactotroph adenomas. ${ }^{16}$ The male DRD2 null mice develop prolactinomas with no background of hyperplasia. These results suggest complex molecular mechanisms involved in the development of prolactinomas, and that decreased dopamine tone in the appropriate endocrine milieu can result in solitary adenomas.

In a previous study, excess homozygosity for polymorphism I in exon 7 of the DRD2 gene was detected in subjects with hyerprolactinemia. An increased frequency of this allele suggests that they are at increased risk for developing hyperprolactinemia. ${ }^{17}$ 
Previous studies of lactotrophs from prolactinomas have found normal DRD2 receptors. A molecular defect in a post-receptor signaling mechanism, such as an somatic inactivating mutation in a Gi protein, could result in autonomous function of the lactotroph resulting in early hyperplasia followed by adenoma formation. ${ }^{18,19}$ Mutations resulting in different receptor $G$ protein interactions, such as Gs (stimulatory) instead of $\mathrm{Gi}$, could result in autonomous lactotroph function. Another explanation would be a linkage between the DRD2 polymorphism I and alterations in MEN-1 gene expression.

Familial inheritance of pituitary adenomas has been described and can be ascribed many times to MEN1. MEN-1 is inherited as an autosomal dominant trait and the affected individuals have anterior pituitary, parathyroid, and pancreatic tumors. Recent studies have localized the MEN-1 gene to $11 \mathrm{q} 13 .{ }^{20,21}$ A kindred from Newfoundland with MEN-1, in which the affected individuals develop prolactinomas, carcinoids, and parathyroid tumors, is due to a nonsense mutation in the MEN-1 gene. ${ }^{22}$ However, in most sporadic pituitary adenomas, mutations of MEN-1 or allelic loss of $11 \mathrm{q} 13$ are not common mechanisms for these tumors. ${ }^{23}$

\section{Treatment}

In patients with hyperprolactinemia the treatment varies with the presenting complaint and the etiology of hypersecretion. In the patient who is on neuropsychiatric medications with antidopaminergic activity, withdrawing the medication will allow prolactin to return to normal in many cases. Withdrawing the offending medication is not an option with many patients due to the severity of the underlying disease. In these subjects one must consider observation or dopaminergic agonists.

In patients with primary hypothyroidism, treatment with thyroxine and normalization of thyroidstimulating hormone levels will normalize prolactin secretion.

In patients with large pituitary lesions that are compressing the stalk, disrupting dopamine transfer, resulting in hyperprolactinemia, the treatment depends on the etiology of the lesion. Granulomas caused by sarcoid may respond to glucocorticoid administration. Other pituitary lesions may require surgery or radiotherapy.

The treatment of prolactinomas depends upon the patient's signs, symptoms, laboratory data, imaging studies, and patient desires. There are a number of reasons to actively treat a prolactinoma including infertility, hypogonadism, and concern about the effects on health primarily the skeleton, significant galactorrhea, or a symptomatic macroadenoma. The treatment of prolactinomas includes medical, surgical, radiation, observation, and combination of these various therapies. The primary treatment for a prolactinoma is medical with a dopamine agonist. If this therapy is ineffective or if the adenoma is cosecreting other pituitary hormones, then surgery or radiotherapy may be warranted.

The dopamine agonist bromocriptine, an ergot derivative, has the longest history and most experience. In subjects with hyperprolactinemia the elevated prolactin levels are rapidly lowered by using dopamine agonists. Not only does bromocriptine decrease prolactin synthesis and release, but it also reduces the size of the pituitary adenoma. Initially, bromocriptine is started at a low dose, such as $1.25 \mathrm{mg}$ daily, and the dose is slowly adjusted upward until therapeutic efficiency is documented. Approximately $60-100 \%$ of patients will normalize prolactin while on bromocriptine. In women, $64-100 \%$ will begin to ovulate and $57-100 \%$ will have cessation of galactorrhea on medical therapy. In 70-90\% of patients the adenomas will shrink while on therapy. Approximately $10-25 \%$ of hyperprolactinemic patients will not respond sufficiently to bromocriptine. These bromocriptine resistant tumors are interesting in their molecular biology with decreased expression of the dopamine D2 receptor isoforms, and no change in spiroperidol-binding sites while on therapy. There have been a large number of different dopamine agonists developed to try and improve tolerability and decrease resistance. The two most commonly used dopamine agonists are cabergoline and pergolide. ${ }^{24,25}$

Surgery and radiotherapy still play a role in the management of hyperprolactinemia. Surgery is helpful in the treatment of patients for debulking a large macroadenoma, the improvement of visual fields, in patients with neurological symptoms, and those nonresponsive to medical therapy. Radiotherapy is usually limited to patients with a macroadenoma that is unresponsive to therapy, in conjunction with medical therapy, or in patients with a macroadenoma unsuccessfully treated with surgery. With the development of a number of dopamine agonists, the need for surgery and radiotherapy has been significantly reduced.

Prolactinomas are one of the most common pituitary tumors. Understanding the molecular biology of these tumors will help to advance the diagnosis and management of these patients. 


\section{References}

1. Ben-Jonathan N, "Regulation of prolactin secretion", in Imwatt (ed), The pituitary gland 2nd ed, New York, Raven Press, (1994): pp. 261-283.

2. Yan G-Z, Pan $W T$, Bancroft $C$, "Thyrotropin-releasing hormone action on the prolactin promoter is mediated by the POU protein Pit-1”, Mol Endocrinol (1991);5: p. 535.

3. Del Pozo E, Wyss H, Tolis G et al., Prolactin and deficient luteal function", Obstet Gynecol (1979);53: p. 283.

4. Wardlaw S L, Bilezikian J P, "Hyperprolactinemia and osteopenia", J Clin Endocrinol Metab (1992);75: p.690.

5. Cartrer F N, Tyson J E, Tolis G et al., "Prolactin-secreting tumors and hypogonadism in 22 men", N Eng J Med (1978);299: p. 847.

6. Kemmann E, Jones J B, "Hyperprolactinemia and headaches", Am J Obstet Gynecol (1983);145: p. 668.

7. Stjean E, Blain F, Comtois R, "High prolactin levels may be missed by immunoradiometric assay in patients with macroprolactinomas”, Clin Endocrinol (1996);44: p. 305.

8. Tritos $N A$, Guay $A$ T, Malarkey $W$ B, “Asymptomatic 'big' hyperprolactinemia in two men with pituitary adenomas”, Eur J Endocrinol (1998);132: p. 82.

9. Reavley S, Fisher A D, Owen D et al., "Psychological distress in patients with hyperprolactinemia", Clin Endocrinol (1997); 47: p. 343.

10. Neill J D, "Prolactin secretion and its control", in Knobil Z, Neill J D (eds), The physiology of reproduction, New York, Raven (1988): pp. 1,379-1,390.

11. Terada T, Kovacs K, Stefaneanu L, Horvath E, "Incidence, pathology and recurrence of pituitary adenomas: Study of 647 unselected surgical cases”, Endocr Pathol (1995);6: p. 301.

12. Martin T L, Kim M, Malarkey W B, "The natural history of idiopathic hyperprolactinemia", J Clin Endocrinol Metab (1985);60: p. 855.

13. Rand T, Kink E, Sator $M$ et al., "MRI of microadenomas in patients with hyperprolactinemia", Neuroradiology (1996)38: p. 744.

14. Caccavelli L, Cussac D, Pellegrini I, Audinot V, Jaquet P, Enjalbert A, "D3 dopaminergic receptors: normal and abnormal transduction mechanisms", Horm Res (1992)38: p. 78.

15. Kelly M A, Rubinstein M, Asa S L, Zhang G, Saez C, Bunzow J R, Allen R G, Hnasko R et al., "Pituitary lactotroph hyperplasia and chronic hyperprolactinemia in dopamine D2 receptor-deficient mice”, Neuron (1997)19: p. 103.

16. Asa S L, Kelly M A, Grandy D K, Low MJ, "Pituitary lactotroph adenomas develop after prolonged lactotroph hyperplasia in dopamine D2 receptor deficient mice”, Endocrinology (1999);140: p. 5,348.

17. Hansen $K A$, Zhang Y, Colver $R$ et al., "The dopamine receptor D2 genotype is associated with hyperprolactinemia", Fertil Steril (2005);84: p. 711.

18. Kreitman M, "Nucleotide polymorphism at the alcohol dehydrogenase locus of Drosophila melanogaster", Nature (1983);304:p. 412.

19. Cronin MJ, Myers G, MacLeod R M, Hewlett E L, "Pertussis toxin uncouples dopamine agonist inhibition of prolactin release", Am J Physiol (1983);244: p. 449.

20. Senogles S E, Benovic J L, Amlacky N, Virson C, Milligan G, Vinistsky R, Spiegel A M Caron M G, "The D2 dopamine receptor of anterior pituitary is functionally associated with a pertussis toxin sensitive guanine nucleotide binding protein", J Biol Chem (1987);262: p. 4,860.

21. Larsson C, Skogreid B, Oberg $K$ et al., "Multiple endocrine neoplasia type I gene maps to chromosome 11 and is lost in insulinoma”, Nature (1988);332: p. 85.

22. Chandrase K, Harappa S C, Garu S C, Manickam P et al., "Positional cloning of the gene for multiple endocrine neoplasia-type I”, Science (1997);276: p. 404.

23. Olufemi S E, Green J S, Manickam P, "Common ancestral mutation in the MEN1 gene is likely responsible for the prolactinoma variant of MEN 1 (MEN1Burin) in four kindreds from Newfoundland”, Hum Mutat (1998);11: p. 264.

24. Kleinberg D L, "Pharmacological therapies and surgical options in the treatment of hyperprolactinemia", Endocrinologist (1997); 7: p. 379.

25. Klibanski A, "Indications for treatment of hyperprolactinemia: an overview", Endocrinologist (1997); 7: p. 376. 